Methods Quantitative real time-PCR data showed that the expression of miR-15a and miR-15b was upregulated in C57BL/6 mice heart subjected to $I / R(n=7)$ while miR-16 without any change, especially miR-15b showed an increase in expression $>3$-fold. And the data was consistent with cardiomyocytes exposed to hypoxia/ reoxygenation $(H / R)(n=3)$. Recombinant adenoviral vectors were constructed to explore the functional role of miR-15b in cultured cardiomyocytes exposed to $\mathrm{H} / \mathrm{R}$. Overexpression of miR-15b enhanced cell apoptosis and the loss of mitochondrial membrane potential $\left(\Delta \psi_{\mathrm{m}}\right)$ determined by flow cytometry analysis. Conversely, downexpression was cytoprotective. Furthermore, the inhibition of miR-15b increased the expression of $\mathrm{Bcl}-2$ protein, suppressed the release of mitochondrial cytochrome $c$ (Cyt-c) to cytosol and decreased the activity of caspase- 3 and caspase- 9 .

Results Quantitative real time-PCR data showed that the expression of miR-15a and miR-15b was upregulated in C57BL/6 mice heart subjected to $I / R(n=7)$ while miR-16 without any change, especially miR-15b showed an increase in expression $>3$-fold. And the data was consistent with cardiomyocytes exposed to hypoxia/ reoxygenation $(H / R)(n=3)$. Recombinant adenoviral vectors were constructed to explore the functional role of miR-15b in cultured cardiomyocytes exposed to $H / R$. Overexpression of miR-15b enhanced cell apoptosis and the loss of mitochondrial membrane potential $\left(\Delta \psi_{\mathrm{m}}\right)$ determined by flow cytometry analysis. Conversely, downexpression was cytoprotective. Furthermore, the inhibition of miR-15b increased the expression of $\mathrm{Bcl}-2$ protein, suppressed the release of mitochondrial cytochrome c (Cyt-c) to cytosol and decreased the activity of caspase-3 and caspase- 9 .

Conclusions MiR-15b may be the upstream regulator of mitochondrial signalling pathway of $\mathrm{I} / \mathrm{R}$ induced apoptosis by targeting $\mathrm{Bcl}-2$.

MiRNAs expression was analysed in $\mathrm{I} / \mathrm{R}$ and sham group (or $\mathrm{H} / \mathrm{R}$ and control group) by using quantitative real-time PCR. Furthermore, gain of function and loss of function methods were employed to investigate the functional roles of miR-15b.

\section{GW23-e2685 \\ MICRORNA-15B IS IMPLICATED IN REGULATING MYOCARDIAL REPERFUSION INJURY BY PROMOTING APOPTOSIS}

\author{
doi:10.1136/heartjnl-2012-302920af.13
}

Lifeng Liu, Zhuo Liang, Xiuhua Liu, L V Zhenrong, Jing Bai, Jie Chen, Yiru Wang, Chen Chen, Yu Wang, Yu Wang. Department of Institute of Geriatric Cardiology

Objectives Myocardial ischaemia reperfusion (I/R) could induce altered expression of microRNAs (miRNAs), which served as powerful regulators of gene expression. Cancer studies have indicated that miR-15a, miR-15b and miR-16 have a potential relationship with apoptosis. The present study was aimed to reveal the roles of above miRNAs for the first time in myocardial I/R injury induced apoptosis. 